\title{
ACCIÓN DEL PIBID BIOLOGÍA EN LA EDUCACIÓN BÁSICA A TRAVÉS DEL ENFOQUE TEMÁTICO FREIREANO: ACCIONES FORMATIVAS EN EDUCACIÓN AMBIENTAL
}

\section{Actions of the Biology PIBID in Basic Education through Freire's Thematic Approach: Educational Actions in Environmental Education}

\section{Ação do PIBID Biologia na Educação Básica através da abordagem temática Feireana: ações formativas na educação ambiental}

\author{
Juliana Rezende Torres ${ }^{1}$ \\ William Mendes Narvona ${ }^{2}$ \\ Fernanda Mizuguchi Leite ${ }^{3}$ \\ Everton Camargo Oliveira ${ }^{4}$ \\ Felipe Gueiros ${ }^{5}$ \\ Augusto César Guilherme Mega ${ }^{6}$ \\ Priscila dos Reis Valentin ${ }^{7}$
}

Fecha de recepción: 12 de agosto de 2016

Fecha de aceptación: 9 de junio de 2017

\section{Resumen}

El presente artículo consiste en un relato de experiencia de corte reflexivo en torno a un proceso de formación de profesores y alumnos viabilizado por un programa del gobierno federal brasileño, el Programa Institucional de Beca de Iniciación a la Docencia (PIBID), a través del establecimiento de la asociación entre universidades y escuelas de educación básica. En este caso, el equipo del PIBID diagnosticó la necesidad de conocer la realidad vivida por los alumnos de la Escuela Estadual Profesor Benedicto Leme Vieira Neto (Salto de Pirapora-São Paulo, Brasil) y sus concepciones sobre el ambiente que les rodea. Este estudio tiene como objetivo describir y analizar críticamente el proceso de formación que se desarrolló entre 2014 y 2015, a través de la metodología de investigación-acción denominada abordaje temático freireano y de un cuestionario cualitativo aplicado a dos alumnos de cada

1 Doctora en Educación Científica y Tecnológica, profesora efectiva del Departamento de Ciencias de Humanidades y Educación de la Universidad Federal de São Carlos, São Paulo, Brasil, julianart@ufscar.br.

2 Licenciado en Educación Física, supervisor del PIBID en la Escuela Estadual Profesor Benedicto Leme Vieira Neto (Salto de Pirapora - São Paulo - Brasil), william_amlliwi@yahoo.com.br

3 Estudiante del Curso de Ciencias Biológicas, UFSCAR Sorocaba, São Paulo, Brasil, fernanda.mleite@live.com

4 Licenciado en Ciencias Biológicas, UfSCAR Sorocaba, São Paulo, Brasil, evertonufscar@hotmail.com

5 Estudiante del Curso de Ciencias Biológicas, ufsCAR Sorocaba, São Paulo, Brasil, feliperaul@gmail.com

6 Estudiante del Curso de Ciencias Biológicas, UFSCAR Sorocaba, São Paulo, Brasil, augustogmega@hotmail.com

7 Licenciada en Ciencias Biológicas, UFSCAR Sorocaba, São Paulo, Brasil, priscila.dosreis@hotmail.com 
aula, en un total de 33 cuestionarios respondidos. Se evaluó cada respuesta y se obtuvieron las siguientes situaciones limitantes de mejores condiciones de vida: problemas ambientales, consecuentes riesgos a la salud de la comunidad, descontento con los ambientes destinados al ocio, falta de identificación con el medio ambiente escolar y sus alrededores. La identificación de estas situaciones permitió la planificación y desarrollo de actividades de formación en la escuela, con el fin de articular los profesores en la educación superior, la educación básica y la formación inicial. Se problematizó la noción de que los casos observados no son individuales y afecta a todos. Como resultado fue observado el impacto positivo de las actividades formativas propiciadas por el equipo PIBID, junto a la comunidad de la escuela, y en el desarrollo de talleres pedagógicos para la continuidad del trabajo.

Palabras clave: Enseñanza de Biología; Educación Ambiental y Salud; Formación de profesores

\section{Abstract}

This paper shares the experience of reflective thinking about a training process for teachers and students that was made viable by a program of the Brazilian federal government, the Institutional Program for Scholarships for Initiation in Teaching (PIBID, for its initials in Portuguese) through a partnership between universities and basic education schools. In this case, the PIBID team diagnosed the need to know the reality of students of the Profesor Benedicto Leme Vieira Neto State School (Salto de Pirapora, São Paulo, Brazil) and their views about their surroundings. The purpose of this study is to describe and analyze, from a critical standpoint, the education process that took place between 2014 and 2015 through the action-research approach known as Freire's thematic approach and a qualitative questionnaire answered by two students from each classroom, for a total of 33 answered questionnaires. Each response was evaluated, and the following situations that limit better living conditions were obtained: environmental problems, consequent risks to the health of the community, dissatisfaction with the environments intended for leisure, lack of identification with the school environment and its surroundings. By identifying these situations, we were able to plan and develop training activities at school, with the aim of articulating teachers in higher education, basic education and initial training. We problematized the notion that the problems observed are not individual and that they affect everyone. As a result, we observed the positive impact of the training activities promoted by the PIBID team and the school community, and on the development of pedagogical workshops to continue the work.

Keywords: Teaching biology; environmental education and health; teacher training

\section{Resumo}

O presente artigo consiste em um relato de experiência de cunho reflexivo sobre o processo de formação de professores e alunos viabilizado por um programa do governo federal brasileiro - PIBID (programa institucional de bolsa de iniciação à docência)- Através do estabelecimento da associação entre universidades e escolas de Educação Básica. Neste caso, a equipe do PIBID diagnosticou a necessidade de conhecer a realidade vivenciada pelos alunos da Escola Estadual Professor Benedicto Leme Vieira Neto (Salto de Pirapora, São Paulo, Brasil) e suas concepções sobre o ambiente que os rodeia. Este estudo tem como objetivo descrever e analisar criticamente o processo de formação desenvolvido entre 2014 e 2015, através da metodologia de pesquisa-ação denominada abordagem temática Feireana e um questionário qualitativo aplicado a dois alunos de cada sala de aula, em total 33 questionários respondidos. Avaliou-se cada resposta e obtiveram-se as seguintes situações limitantes de melhores condições de vida: problemas ambientais, consequentes riscos à saúde da comunidade, desconformidade com os ambientes destinados ao lazer, falta de identificação como o ambiente escolar e suas proximidades. A identificação dessas situações possibilitou a planificação e o desenvolvimento de atividades de formação na escola, visando articular os professores na educação superior, o ensino básico e a formação inicial. Problematizou-se a noção de que os problemas observados não são individuais e a afetam à comunidade. Como resultado, evidenciou-se o impacto positivo das atividades formativas propiciadas pela equipe PIBID, em parceria com a comunidade da escola, no desenvolvimento de oficinas pedagógicas para a continuidade do trabalho.

Palavras-chave: ensino de biologia; educação ambiental e saúde; formação de professores 


\section{Introducción}

El PIBID es una iniciativa del Gobierno federal brasileño (Ministerio de Educación) financiada por la Coordinación de Perfeccionamiento de Personal de Educación Superior (CAPES). Consiste en la vinculación entre las universidades y las escuelas públicas, con el objetivo de contribuir a la iniciación en la enseñanza de licenciados, futuros maestros de educación básica. En este contexto, todos los involucrados en el programa son eruditos PIBID, con la siguiente estructura interna: un coordinador institucional (para la universidad), los coordinadores de zona (académicos en las áreas de conocimiento), supervisores (profesores de educación básica) y licenciados (estudiantes de licenciaturas en proceso de formación inicial). Así, el PIBID es una iniciativa para la mejora y la valorización de la formación del profesorado de educación básica, con el objetivo principal de traer los licenciandos a la realidad del entorno escolar de la enseñanza pública, en una perspectiva de iniciación a la enseñanza para mejorar la calidad de la educación pública. Además del carácter educativo del PIBID, destacamos la fragilidad que gira en torno al mantenimiento de lo mismo, en el momento histórico y político actual que atraviesa el país, en vista de que la PIBID aún no se ha convertido en una política pública oficial. En este sentido, es importante destacar que existe en el país un distanciamiento entre la proposición y la formalización de las políticas públicas educativas y la formación del profesorado, in locus.

A través del acercamiento entre la universidad y la escuela promovida por el PIBID, ha sido posible lograr prácticas educativas en las escuelas públicas de diferentes ciudades brasileñas, incluyendo Salto de Pirapora (región administrativa de Sorocaba). En esta región hubo un vínculo entre el campus de la Universidad Federal de São Carlos Sorocaba (Sorocaba ufscar) y algunas escuelas, entre ellas, la Escuela Estadual Prof. Benedicto Leme Vieira Neto, en Salto de Pirapora, São Paulo.

La escuela está ubicada en una región de la periferia, atiende a estudiantes de cuatro barrios de la ciudad, que en su mayoría corresponden a las clases media y baja. Ofrece primaria y secundaria en tres turnos y es una facultad de profesores efectivos y sustitutos ${ }^{8}$. La escuela tiene una gestión abierta al desarrollo de proyectos innovadores y un equipo participativo, impulsado por la mejora de la calidad de las escuelas.

8 Profesor efectivo: profesor oficial con relación de empleo. Profesor sustituto: profesor contratado por un periodo temporario, sin relación de empleo.
En este contexto, en marzo de 2014, comenzaron las actividades PIBID en la escuela a través de las acciones desarrolladas de cuatro subproyectos: Biología, Química, Física e Interdisciplinar. Lo que representa un total de cuatro profesores coordinadores, de ocho profesores supervisores y cuarenta y un académicos licenciados con becas. En este trabajo describimos las acciones formativas dentro del subproyecto de biología, tres grupos, cada uno de ellos compuesto por un maestro de educación básica y cinco licenciados en ciencias biológicas, cada uno con un enfoque temático, bajo la coordinación de un miembro de la Sorocaba ufscar.

Las acciones iniciales de este subproyecto en la escuela fueron la realización de una investigación cultural con estudiantes y otros integrantes de la escuela, dirigida a identificar las situaciones principales limitantes de mejores condiciones de las escuelas de vida y de la comunidad local. Basado en las situaciones que fueron planteadas por el proceso de investigación activado, surgió el nombre del grupo: Medio Ambiente y Salud, cuyas actividades se describen a continuación. De hecho, este trabajo tiene como objetivo la clarificación y reflexión crítica de las acciones formativas desarrolladas por este grupo PIBID - Biología, junto con la escuela pública.

\section{Metodología}

El trabajo del grupo en la escuela se basó en los fundamentos y procedimientos teórico- metodológicos de la dinámica del enfoque temático freireano (Delizoicov, Angotti y Pernambuco, 2002), que fue sistematizado en cinco pasos del proceso de investigación temática descrita en el capítulo 3 de la obra Pedagogía del oprimido (Freire, 1987). Esta dinámica educativa consiste en desarrollar programas escolares críticos basados en contradicciones sociales o situaciones límite existenciales, que, a su vez, se obtienen mediante la realización de una investigación sociocultural en la comunidad donde se encuentra la escuela. Así, las acciones formativas desarrolladas en la escuela siguieron los pasos del enfoque temático freireano:

Primer paso: “levantamiento preliminar" (Freire, 1975, p. 122):

Este es el momento en el cual se realiza un estudio de las condiciones de la localidad. A través de datos escritos e informales conversaciones con personas (estudiantes, padres, representantes de asociaciones, entre otros), visitas y observaciones de organismos públicos (como los centros de asistencia de salud, hospitales, mercados, etc.) y el uso de cuestionarios, 
una recopilación de datos. (Delizoicov, 2008, p. 48, traducción propia).

El material recogido puede organizarse en la forma de un expediente, incluyendo información, testimonios, fotos, videos, diarios de campo, datos estadísticos, datos históricos, acciones institucionales existentes o programados; con el fin de contemplar la suma de toda clase de datos, información de registro e impresiones grabadas. El análisis colectivo de estos datos e información permitirá la construcción de un grupo escolar y construir "categorías" de una manera más flexible, no estática, así se pueden interpretar a menudo, modificarlos o descartarlos. Este expediente debe estar abierto a nuevos registros, los nuevos análisis y los registros resultantes de la acción educativa (São Paulo, 1990, citado por Torres, 2010).

Segundo paso: "Análisis de las situaciones y la elección de las codificaciones" (Freire, 1975, p. 126), "es la etapa cuando, basándose en el análisis de los datos agarrados, se hace la selección de las situaciones que contienen las contradicciones vividas y la preparación de sus codificaciones, que se presentará en el próximo paso" (Delizoicov, 2008, p. 48, traducción propia).

Aquí, los fenómenos y situaciones relevantes y significativos en la vida de la población investigada comienzan a ser identificados. De esta manera, los educadores, usando ahora entrenamientos diferenciados, analizan el material recogido, tratando de encontrar las relaciones entre las palabras que expresan la visión de la población en un intento por encontrar lo que es significativo para este grupo social, percibido por ellos como una dificultad por superar y, al mismo tiempo, la posibilidad de comprender el contexto más amplio en su realidad, y lo que caracteriza el generador del tema que resume las contradicciones sociales vividas.

De esta manera, las posibles situaciones significativas, de las cuales surgirán los temas generadores, se plantean por medio de un proceso de análisis cualitativo que consiste en la construcción de categorías de análisis, entendidas como un instrumento de trabajo del investigador participante. Estas categorías son un tipo de codificación que permite un grupo más amplio de informaciones obtenidas, como su naturaleza, por lo que se recogieron los ángulos más significativos de las situaciones observadas (São Paulo, 1990).

Tercer paso: "Diálogos decodificadores" (Freire, 1987): Paso que coincide con lo que Freire (1987) llama círculo de investigación temática, que consiste en las reuniones que tienen como objetivo la validación de situaciones específicas y los temas representados en las codificaciones, como hecho significativo para la población. Esta validación se produce desde la presentación de las codificaciones (que representan las contradicciones sociales de la realidad investigada) al grupo más amplio de los estudiantes (y sus familias), que son criados mediante un proceso dialógico, encaminado a la decodificación de estas situaciones para confirmar o no los temas.

Por lo tanto, es posible entender el generador del tema como:

Un objeto de estudio que comprende el hacer y pensar, acto y reflexionar, teoría y práctica. En este sentido, supone un estudio de la realidad de la que surgirá una red de relaciones entre situaciones significativas (una dimensión significativa de la individual, social e histórica) y una red de relaciones que guía la discusión de la interpretación y representación de esa realidad. Por su naturaleza el tema asume también una visión de totalidad y comprensión de esta realidad y la ruptura de los conocimientos a nivel de sentido común, una vez señalando el límite de la comprensión qu e la comunidad tiene sobre esta realidad. El generador del tema, por lo tanto, presupone la superación de este límite. (São Paulo, 1991, p. 8).

Cuarto paso: "Reducción temática" (Freire, 1987): Paso que representa un desafío para la comprensión de los temas y tiene como objetivo planificar su enfoque en el proceso educativo. Aquí comienza el proceso de reducción de los temas por expertos en su campo, que consiste en la selección de contenidos específicos por criterios pedagógicos y epistemológicos, que formará parte del currículum escolar. Como señala Freire, el experto debe:

Introducir el grupo interdisciplinario el proyecto de 'reducción' de su tema. En el caso de "reducción” de esto, la búsqueda experta sus núcleos fundamentales, constituyéndose en las unidades de aprendizaje y establecer una secuencia entre sí mismos, dan el resumen del tema "reducido". (1987, p. 115).

Según Pernambuco (1994), los posibles temas son vistos bajo la óptica de todos los temas del currículo escolar, teniendo en cuenta las expresiones obtenidas sobre ellos en los círculos de investigación temática, con el objetivo de la unión entre los diferentes puntos de vista. A partir de ahí, los resultados son ordenados, conforme el grupo de edad y la información disponible sobre las posibilidades de cada clase graduanda, además de los principios de estructuración de cada disciplina. La secuencia de los resultados de la reducción de algunos temas seleccionados significa buscar relacionar a los 
temas reducidos, los respectivos paradigmas o teorías científicas pertinentes a ellos, que, alternadamente, constituirán los contenidos específicos subyacentes a las actividades educativas previstas en la programación.

Quinto paso: "Sala de aula" (Freire, 1987): Este paso corresponde a círculos de cultura (Freire, 1987) llevados a cabo en cursos de alfabetización de adultos, por Freire. Según Delizoicov (2008), solo después de los cuatro pasos anteriores, dirigidos a la obtención, estudio y planificación de temas por el equipo interdisciplinario, es que se reanuda la acción educativa, en un paso más ampliado, con el programa establecido y los materiales didácticos pedagógicos para ser utilizado con los alumnos, ya sistematizado.

\section{Resultados y discusión: acciones formativas en la escuela}

Considerando que las acciones formativas desarrolladas en la escuela trataron de seguir los procedimientos teóricos y metodológicos del enfoque dinámico de la temática educativa freireana, se describió y analizó críticamente el proceso desarrollado durante el 2014 y el 2015, por el grupo de biología de PIBID, Medio Ambiente y Salud, en la Escuela Estadual Benedicto Leme Vieira Neto.

\section{1. ${ }^{a}$ fase del enfoque temático freireano: "Encuesta preliminar de la realidad local"}

En el supuesto de que el PIBID opera dentro de las escuelas públicas con el fin de promover una problematización circunstancial de la escuela y también el sitio donde se inserta el mismo, fue reconocido por el grupo la necesidad de conocer la realidad vivida por los alumnos de la escuela pública profesor Benedicto Leme Vieira Neto, así como elevar los conceptos de ellos sobre el entorno que les rodea. Para ello, la medida adoptada fue la elaboración de un cuestionario cualitativo que contiene 12 preguntas, aplicado a dos alumnos de cada clase en los tres turnos del funcionamiento de la escuela, con un total de 33 cuestionarios respondidos. Evaluaron cada respuesta cuidadosamente y discutieron los resultados, con el fin de llegar a un diagnóstico inicial. Entre los aspectos planteados, es evidente que los estudiantes no están contentos con el barrio en que residen, y señalan como factores negativos la acumulación de basura en las calles provocando mal olor en los ríos y arroyos que recorren el barrio, así como la falta de conservación del medio ambiente. La mayoría de las respuestas también señalaron la falta de lugares de ocio y cultura dentro del barrio, así que las actividades recreativas que realizan son los partidos de fútbol en una cancha local y juegos en las plazas, que se conservan en su mayoría en mal estado. Fueron señalados también problemas de infraestructura básica como calles llenas de baches y la acumulación de malas hierbas en lotes baldíos y áreas recreativas.

Un factor importante de la participación de los estudiantes en las actividades de barrio es que no son (o son pocas) ofrecidas actividades culturales o de ocio a estos residentes. Entre ellas, un programa público es Escuela de Familia, programado los fines de semana en la escuela, que consiste en algunos talleres de artesanía, juegos y capoeira. Además, el distrito no tiene ninguna asociación de la comunidad de residentes y estudiantes, por lo que subrayaron la necesidad de que esto se produzca con una participación más activa y conjunta para apoyar mejoras en la comunidad.

\section{2. a fase del enfoque temático freireano: "Análisis de situaciones y decisiones de las codificaciones"}

En este paso comenzamos a identificar fenómenos y situaciones relevantes y significativas en la vida de la población. Durante este proceso, a través de una formación diferenciada, se revisó todo el material recogido en el cuestionario, con el propósito de encontrar las relaciones entre las líneas donde los estudiantes expresaban la visión de la población, en un intento por obtener algo significativo para el grupo social, es decir, asumimos la labor de encontrar algo señalado por los estudiantes como una gran dificultad a superar para poder entender también en un contexto más amplio la realidad en la que se encuentran.

El grupo reunido mediante el análisis de las líneas de los cuestionarios, es decir, las situaciones límites de mejores condiciones de vida para la comunidad escolar de Escuela Estadual Profesor Benedicto Leme Vieira Neto, presentó los siguientes aspectos: a) los problemas ambientales, tales como la contaminación de ríos y zonas verdes; b) riesgos para la salud que amenazan la comunidad; c) inconformidad con los espacios destinados al ocio, debido a la falta de estructura y problemas con los residuos urbanos; d) falta de identificación de estudiantes con el entorno escolar y sus alrededores. Basado en estos supuestos, situaciones límite (Freire, 1987), posibles contradicciones vividas por la comunidad local, el siguiente paso fueron las opciones de codificación (códigos) que representasen las principales situaciones que enfrenta la comunidad, con el objetivo de legitimarlos o no como contradicciones por ellos vividas, así como elevar sus entendimientos y límites explicativos sobre 
situaciones sintetizadas por el grupo. Así comenzaron las acciones formativas del equipo PIBID-Biología en la escuela mediante el proceso codificación-problematización-descodificación (Freire, 1987) en la dirección indicada arriba. Fueron codificadas y planteadas, junto a los estudiantes (y la comunidad), tres actividades en torno a la posibilidad de las situaciones límite diagnosticadas por el grupo: 1) panel con imágenes y frases para la escena problemática de la basura; 2) organización de habitación temática con la ruta de la basura; 3) visita monitoreada al vertedero de basura y reciclaje de cooperativa en el municipio de Salto de Pirapora.

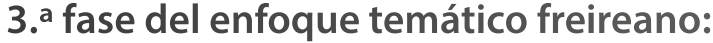 "Diálogos decodificadores"}

Este paso corresponde al círculo de investigación temática (Freire, 1987), con el propósito específico de validación de situaciones y contradicciones que estuvieron representadas en las codificaciones, significado, al frente de la población.

En la actividad 1, se optó por fotografiar los alrededores de la escuela y el vecindario, se enfocó, sobre todo, la disposición de la basura, las plazas y el parque público ubicado junto a la escuela, en el cual fluye el río Salto de Pirapora. Las fotos fueron expuestas en un mural en el patio de la escuela con frases que generan reflexión e impacto; el grupo se dividió para cumplir con los tres turnos de las escuelas, y preguntaron a los estudiantes acerca de los lugares fotografiados y la situación en la que se encuentran debido a la basura lanzada en la comunidad donde residen (¿sabes cuál es este lugar? ¿Dónde estas fotos fueron tomadas? ¿Cuál es su posición sobre estas imágenes?). Durante la sesión, los estudiantes registraron sus concepciones sobre el contenido de las imágenes y frases en uno de los paneles de la izquierda en el patio.

Antes de dicha acción, nos dimos cuenta de que los estudiantes identificaron los lugares fotografiados, pero les daba vergüenza frente a sus colegas. Algunas opiniones vinieron a legitimar las cuestiones presentadas: "Creo que si cada uno se educa sería mucho mejor y menos sucio" y "conozco la mayoría de los lugares fotografiados, todo esto es consecuencia de la falta de conciencia (conocimiento) de la sociedad". Por lo tanto, se evidenció que los estudiantes reconocen tales situaciones como problemático en la comunidad.

En la actividad 2, se produjo la construcción del "Camino de la basura", una ruta con información (fotos, frases y "basura"), expuesta en un aula, que describe todas las etapas de producción de los residuos, desde su fabricación hasta su destino final. La acción se llevó a cabo en el contexto de un proyecto de la escuela: "Un día en la escuela de mi hijo", que ocurre dos veces al año, cuando la escuela abre sus puertas un fin de semana para la comunidad en general con diferentes actividades. La propuesta de crear un túnel interactivo es llevar a los estudiantes y la comunidad la idea de cómo es la producción de una botella de PET y su destino final. El túnel se basó en seis etapas, cada miembro del grupo quedó responsable de un enfoque en lo referente a la basura: 1) producción de una botella de PET (cómo se produce, cuáles son los materiales utilizados, su ruta después de ser producidos en las fábricas, pasando por la industria compradora, donde usted recibirá su contenido para la venta); 2) la compra de ese producto en el comercio, es decir, cómo la botella PET llega a nuestros hogares; 3) disponer de PEт botella después de consumo: las compañías son responsables de recoger basura en ciertos días de la semana en cada calle de la ciudad; 4) después de recogidos por el camión de basura, ¿cuál es el destino de botella de PET? ¿Los rellenos sanitarios y vertederos? 5) ¿Qué es vertedero y basura, cómo funciona y cuáles son sus consecuencias? ¿Cómo funciona y cuáles son sus consecuencias? ¿Este vertedero es infinito?, 6) final de la trayectoria de basura: ¿la botella de PET estará en ese relleno sanitario o vertedero por cuánto tiempo? ¿Esto podría cambiar?

En todas las etapas se discutieron cuestiones importantes, como las políticas públicas, acción de la sociedad del conocimiento, consumismo, obsolescencia planificada de reciclaje/reutilización de productos. Al salir del túnel, el visitante tuvo la oportunidad de ver fotos de los rellenos sanitarios y vertederos, teniendo en cuenta la cantidad de residuos depositados en estos lugares con imágenes tomadas de internet y expuestas en la pared. El objetivo era causar algún impacto con las imágenes. Desde ese momento, nos preguntamos si este destino podría ser cambiado y cómo.

Tomamos nota durante el camino recorrido, estudiantes y otros miembros de la comunidad al ser cuestionados sobre los aspectos relativos al destino de la basura por ellos descartados, muchos no saben de dónde fueron tirados estos residuos en su ciudad, ni cuáles fueron los procedimientos que permiten su reducción. No sabían si en la ciudad existió algún tipo de colección especial para los residuos reutilizables e incluso negaban la existencia de cualquier tipo de relleno sanitario en la ciudad. En este marco, los resultados adquiridos en esta actividad de problematización y diálogos decodificadores de la legitimación de las contradicciones identificadas por el grupo (en busca de temas generadores para la producción de programas críticos de la escuela) 
es que sugerimos hacer la siguiente actividad, con el fin de propiciar en los estudiantes el conocimiento sobre la realidad del destino de los residuos producidos por la comunidad de Salto de Pirapora.

En la actividad 3, el grupo articuló una visita supervisada por el secretario de Medio Ambiente de Salto de Pirapora al vertedero y a la cooperativa de reciclaje de la ciudad, contemplando veinte alumnos de la escuela que mostraron interés en participar. Por lo tanto, se llevó a cabo junto a la escuela la visita monitoreada al relleno sanitario ubicado a las afueras de la ciudad, en un ambiente de residencias, con el fin de evitar cualquier tipo de contacto que pueda causar daños a la población. Fue problematizada la manera como se separa esta basura en el vertedero y su proceso de toma de tierra sobre la profundidad y las capas necesarias. Durante la visita a la cooperativa de reciclaje, fue problematizada la forma en que la basura reciclable alcanza a la cooperativa y cuál es el proceso desde la separación manual, hasta su final de prensa, constituyendo de esta manera grandes fardos para la venta.

Fue en el proceso de problematización-descodificación que obtuvimos las visiones que los estudiantes tenían acerca del destino de los residuos producidos por la comunidad. Se observó que los estudiantes comenzaron a mostrar más interés en las situaciones límite que experimentó la comunidad, pues, cuando lograron comprender mejor el contexto de situaciones codificadas y su propia realidad experiencial, los estudiantes comenzaron a entender la importancia del grupo de acciones formativas PIBID-Biología Medio Ambiente y Salud, desarrolladas en la escuela de conciencia ambiental. Durante el año 2015, el grupo trabajó para la obtención/legitimación del tema generador a través de la presentación de un video conimágenes de todala trayectoria del proyecto, que contiene las acciones realizadas dentroy fuera de la escuela y desarrollado en conjunto con los estudiantes algunos botes de basura y pufs con materiales reutilizables recogidos en la comunidad circundante, o que se señalan por ellos, para que continúe con los pasos 4 y 5 , destinadas a la elaboración y desarrollo de los programas escolares mediante la realización de procesos formativos, (re)configuraciones de plan de estudios y actividades didáctico-pedagógicas.

\section{Conclusiones}

En términos generales, este trabajo consiste en un proceso esencialmente formativo, que articula la investigación y acción, teoría y práctica, conocimiento de sentido común y conocimiento sistemático, las contradicciones sociales locales y contradicciones sociales globales.
Se considera que la realización de estas acciones formativas permiten el desarrollo de un proyecto-escuela de educación ambiental crítico-transformador en la escuela (Torres, 2010; Torres, Ferrari y Maestrelli, 2014), cuya articulación está dada por los atributos del campo de la educación ambiental y los fundamentos freireanos de la educación, por lo tanto, su carácter critico-transformador que se presenta como demanda en términos de políticas públicas de educación ambiental en el país. Las perspectivas de continuidad es completar el cuarto paso que configura la reducción temática y consiste en configuraciones curriculares, especialmente en el área de la enseñanza de la biología y el quinto paso es el aula a través del desarrollo de actividades didácticas-pedagógicas destinadas a enseñanza de la ciencia y biología. Con este trabajo hemos desarrollado acciones de formación para la educación ambiental en la escuela, desde una concepción de la educación libertadora y emancipadora y una concepción del ambiente fundada sobre la relación entre sociedad y naturaleza.

\section{Referencias}

Delizoicov, D. (2008), Perspectiva de la educación en ciencias y de Paulo Freire. Alexandria, 1(2), 37-62.

Delizoicov, D., Angotti, J. A. P., y Pernambuco, M. M. (2002). Enseñanza de la ciencia: fundamentos y métodos. São Paulo: Cortez.

Freire, P. (1975). Pedagogía del oprimido. Rio de Janeiro: Paz e Terra.

Freire, P. (1987). Pedagogía del oprimido. Rio de Janeiro: Paz e Terra.

Pernambuco, M. M. (1994). Educación y la escuela como un movimiento de enseñanza de las ciencias a la transformación de la escuela pública (Tesis doctoral en Ciencias de la Educación). Universidad del São Paulo, São Paulo, Brasil.

São Paulo. (1990). Departamento de la Ciudad de la Educación. Estudio preliminar de la realidad local: la recuperación de la vida cotidiana. En: El entrenamiento de los libros. Serie: acción pedagógica en la escuela a través de la interdisciplinariedad. São Paulo: DOT/SME-SP.

São Paulo. (1991). Departamento de la Ciudad de la Educación. Tema generador y la construcción del programa: una nueva relación entre el currículo y la realidad. En: El entrenamiento de los libros. Serie: acción pedagógica en la escuela a través de interdisciplinariedad. São Paulo: DOT/SME-SP. 
Torres, J. R. (2010). Educación ambiental crítico-transformador y enfoque temático freireano (Tesis Doctoral en Educación de Ciencia y Tecnología). Universidad Federal del Santa Catarina, Florianópolis, Brasil.
Torres, J. R., Ferrari, N., y Maestrelli, S. R. P. (2014). Educación ambiental crítico-transformador en lo contexto escolar: teoría y práctica freireana. En C. F. B. Loureiro y J. R. Torres (Eds.), Diálogo de educación ambiental con Paulo Freire. São Paulo: Cortez. 